\title{
Lighting the way
}

\section{The innovation and optimization of optogenetic tools are providing new insights into biological and cellular processes with unparalleled spatiotemporal resolution.}

The field of optogenetics was born out of pioneering work using light-gated ion channels to manipulate the activity of neuronal circuits (F1000 Biol. Rep. 3, $1,2011)$. Subsequent collaborations between chemical and cell biologists have extended these tools to address questions in cell signaling and gene expression in other cell types and tissues. The versatility of light in controlling cellular processes reversibly with spatiotemporal resolution has revolutionized biology, prompting Nature Methods to declare optogenetics the 2010 Method of the Year (Nat. Methods $\mathbf{8}, 1,2011)$. Since then, researchers have been primarily focused on improving the performance of these light-responsive systems or applying them to either mimic or impede biological events ranging from epigenetic regulation to membrane trafficking. Optogenetics is philosophically aligned with chemical biology in its desire to develop versatile tools, apply them to find molecular answers to biological questions, and create biological systems with tailored properties (Nat. Chem. Biol. 10, 165, 2014). This intersection is highlighted by a collection of pieces published recently in Nature Chemical Biology describing new optogenetic strategies and applications that allow the precise dissection of biological processes.

Optogenetic techniques were largely inspired by naturally occurring lightdependent regulatory systems. Most early optogenetic approaches converted three types of photoreceptor proteins (cryptochrome (CRY2), phytochrome (PYHB), and light-oxygen-voltage domain (LOV)) into genetically encoded lightsensitive effectors. Although the initial optogenetic systems were fairly efficient, significant efforts have since been made to optimize light sensitivity and kinetics and to make dark-to-light photoconversion more efficient. For example, insights from structural analyses of these sensors (Nat. Chem. Biol. 10, 801-809, 2014) allowed the engineering of variants with greater dynamic range (Nat. Chem. Biol. 10, 196-202, 2014). In another study, Taslimi et al. performed a yeast selection screen to identify CRY2 mutants that exhibited shorter or longer half-lives upon binding with their interacting partner, CIB1, and thus could be used to modulate the duration of photoactivation (Article, p. 425).

Altogether, the development of variants with altered activity expands the diversity of the optogenetic toolbox for additional applications.

Structural analyses of photoreceptor proteins have defined light-dependent conformational changes that recruit and promote heterodimerization with their binding partners. These observations have inspired various applications that use light-induced complex formation to alter biological activity. For example, the addition of a CRY2-CIBI1 heterodimerization system to the transcription activator-like effector (TALE) (Nature 500, 472-476, 2013) and Cas9 (Nat. Chem. Biol. 11, 198-200, 2015) systems converts these genomeediting proteins into blue-light-mediated transcriptional activators. Nguyen et al. have now used the CRY2-CIB1 system to promote light-mediated aggregation of Rab GTPase family members, resulting in disruption of membrane trafficking (Article, p. 431). Yumerefendi et al. have added a nuclear export signal combined with a LOV2 domain to the E3 ubiquitin ligase Bre1 to move Bre1 out of the nucleus upon blue-light exposure (Brief Communication, p. 399). As these examples illustrate, coupling optogenetic systems to functionally important proteins offers the potential to alter virtually any biological processes with a predetermined wavelength of light.

In addition to using natural photoreceptors, optogenetics has also benefited from the application of synthetic photochromic compounds. One such compound, azobenzene, undergoes lightdependent isomerization between trans and cis conformations, acting as a highly reversible and durable activity switch (Angew. Chem. Int. Ed. Engl. 50, 12156$12182,2011)$. The attachment of azobenzene to small-molecule ligands has been put to great effect to activate ion channels (Nat. Chem. Biol. 2, 47-52, 2006) and G-protein-coupled receptors in cells (Nat. Chem. Biol. 10, 813-815, 2014). Recent efforts combining chemical probes with optogenetics endow the potent and specific modulation of a target's function with spatiotemporal resolution. Replacement of the central carbon-carbon bond of a microtubule inhibitor, combretastatin A-43, with an azobenzene moiety makes it possible to control microtubule dynamics through light exposure in a rapid manner (Cell 162, 403-411, 2015). Reis et al. devised azobenzene-containing histone deacetylase (HDAC) inhibitors that bind and inhibit the activity of selected HDACs with light exposure (Nat. Chem. Biol. 12, 317-323, 2016). Taken together, these approaches provide an orthogonal means for creating light-responsive chemical ligands and associated proteins.

Optogenetics also impacts chemical biology research in the arena of highthroughput screening. Although chemical screening remains one of the best means to identify novel inhibitors of a particular biological target, the process can be labor intensive, prompting a need for more efficient alternatives. Ingles-Prieto et al. demonstrated the potential of optogenetics to simplify small-molecule screening (Nat. Chem. Biol. 11, 952-954, 2015) by facilitating the identification of small molecules that promote or block the activity of a light-inducible orphan receptor. The use of light as the activator for the assay limits the addition of reagents and operational steps, providing a more efficient alternative for chemical screening.

Despite significant recent progress in optogenetic research, countless cellular processes remain hidden in the shadows. At Nature Chemical Biology, we welcome submissions that illuminate these areas by describing new optogenetic tools and methods as well as significant improvements to existing optogenetic systems that convincingly outperform related methods. We also encourage submissions that use optogenetic systems to deliver new mechanistic insights into biological systems or to provide synthetic biology tools for biological engineering applications.

We hope that these papers offer a glimpse of the promise of optogenetic research. We are excited about the progress of this field and eagerly await the application of these tools to reveal new and exciting insights into biological processes that were not previously accessible. 